\title{
The effects of oral nutritional interventions combined with physical activity on nutritional and functional outcomes in the management of malnutrition: a systematic review
}

\author{
H. Ayling and C. Baldwin \\ King's College London, 150 Stamford Street, London, SE1 9NH, UK
}

The effects of oral nutritional interventions on functional and nutritional outcomes in nutritionally vulnerable individuals are inconsistent, thus parallel therapies to enhance therapeutic management warrant exploration ${ }^{(1)}$. Physical activity, shown to attenuate the inflammatory response $^{(2)}$ and enhance appetite ${ }^{(3)}$, is a cost effective and accessible option ${ }^{(4)}$. This systematic review and meta-analysis synthesizes the evidence for nutritional intervention and physical activity combined compared to nutrition alone, exercise alone or routine care in nutritionally vulnerable individuals.

Searches of 5 electronic databases and bibliographies were undertaken to September 2011. Trials comparing oral nutritional intervention and exercise with (1) nutritional intervention alone, (2) exercise alone or (3) routine care were eligible for inclusion. Data on energy intake, weight, fat free mass, strength and gait were entered into a meta-analysis using Review Manager 5.0.

23 randomised controlled trials (RCTs) were identified ( $n=1430$ participants) including elderly (11 RCTs), COPD (2 RCTs), HIV (2 RCTs), critical illness (1 RCT), renal disease (2 RCTs), muscle wasting (1RCT) and osteo-degenerative disease (4 RCTs). Duration of follow up ranged from 7 to 78 weeks. 10 studies compared combined intervention with nutritional alone, 11 with exercise alone and 12 with routine care. 7 studies included comparisons in more than one part of the review. Two studies provided no usable data. One study included personalized dietary counseling, with no food or oral nutritional supplementation, as the nutritional intervention and accounted for all of the heterogeneity in analyses; results are presented with this study removed ${ }^{(3)}$. All studies were judged to be at risk of bias for one or more characteristic.

\begin{tabular}{|c|c|c|c|}
\hline & $\begin{array}{c}\text { Combined intervention } \\
\text { vs. nutrition MD (95\% CI) }\end{array}$ & $\begin{array}{c}\text { Combined intervention } \\
\text { vs. exercise MD (95\% CI) }\end{array}$ & $\begin{array}{l}\text { Combined intervention } \\
\text { vs. routine care MD }(95 \% \mathrm{CI})\end{array}$ \\
\hline Energy intake (MJ) & $0.72(0.58 \text { to } 0.85)^{*}, n=4$ & $1.29(0.83 \text { to } 1.75)^{*}, n=2$ & $1.44(1.03 \text { to } 1.86)^{*}, n=2$ \\
\hline Weight (kg) & $0(-0.52$ to 0.52$), n=5$ & $1.42(0.86 \text { to } 1.98)^{*}, n=5$ & $1.72(0.99 \text { to } 2.44)^{*}, n=2$ \\
\hline Fat free mass $(\mathrm{kg})$ & $0.25(-0.21$ to 0.71$), n=5$ & $-0.44(-1.07$ to 0.19$) n=3$ & $0.67(-1.02$ to 2.37$), n=2 \mathrm{I}^{2}=72 \%$ \\
\hline Strength $(\mathrm{kg})$ & $7.92(-0.23$ to 16.1$), n=5$ & $0.83(-0.45$ to 2.1$), n=6$ & $1.28(-0.43$ to 3$), n=4$ \\
\hline Gait $(\mathrm{m} / \mathrm{s})$ & $0.03(-0$ to 0.07$), n=4$ & $0(-0.03$ to 0.04$), n=3$ & $0.06(0.03 \text { to } 0.1)^{*}, n=4$ \\
\hline
\end{tabular}

$\mathrm{MD}=$ mean difference, $*=$ result statistically significant, $\mathrm{CI}=$ confidence interval, $n=$ number of studies.

Nutritional intervention combined with physical activity is associated with significant improvements in energy intake compared with either therapy alone or routine care and may result in improvements to weight and functional improvements.

Nutritional intervention given in combination with exercise has promising effects on energy intake but larger studies are needed to investigate whether these increases have effects on clinical, functional and patient centred endpoints.

1. Koretz RL, Avenell A, Lipman TO et al. (2007) Am J Gastroenterol 102, 412-29.

2 Gleeson M, McFarlin B and Flynn M (2006) Exerc Immunol Rev 12, 34-53.

3. Stephens NA and Fearon KCH (2008) Medicine 36, 78-81.

4. Roine E, Poine R, Rasanen P et al. (2009) Int J Technol Assess 25, 427-454.

5. Rydwik E, Gustafsson T, Frandin K et al. (2010) Aging-Clinical \& Experimental Research 22, 85-94. 